
\title{
25 Research Square \\ Selective inhibition of microRNA processing by a herpesvirus-encoded microRNA triggers virus reactivation from latency
}

Thomas Hennig

University of Würzburg

Archana Prusty

Julius-Maximilians-University Würzburg

Benedikt Kaufer

Frei University of Berlin

Adam Whisnant

Julius-Maximilians-University Würzburg

Manivel Lodha

Julius-Maximilians-University Würzburg

Antje Enders

Julius-Maximilians-University Würzburg

Julius Thomas

Julius-Maximilians-University Würzburg

Francesca Kasimir

Julius-Maximilians-University Würzburg

Arnhild Grothey

Julius-Maximilians-University Würzburg

Stefanie Herb

Julius-Maximilians-University Würzburg

Christopher Juerges

Institute for Virology and Immunobiology, Julius-Maximilians-University Würzburg https://orcid.org/0000-0001-5617-6601

Gunter Meister

University of Regensburg https://orcid.org/0000-0002-2098-9923

Florian Erhard

University of Wuerzburg https://orcid.org/0000-0002-3574-6983

Lars Dolken ( $\square$ lars.doelken@uni-wuerzburg.de)

Julius-Maximilians-University Würzburg https://orcid.org/0000-0002-4651-3544

Bhupesh Prusty

Julius-Maximilians-University Würzburg 


\section{Biological Sciences - Article}

Keywords: Herpesvirus, HHV-6, miRNA processing, miR-30, mitochondria, fusion and fission, type I interferon, latency, virus reactivation

Posted Date: September 3rd, 2021

DOI: https://doi.org/10.21203/rs.3.rs-820696/v1

License: (c) (i) This work is licensed under a Creative Commons Attribution 4.0 International License. Read Full License

Version of Record: A version of this preprint was published at Nature on May 4th, 2022. See the published version at https://doi.org/10.1038/s41586-022-04667-4. 


\section{Abstract}

Herpesviruses have mastered host cell modulation and immune evasion to augment productive infection, life-long latency and reactivation thereof 1,2. A long appreciated, yet elusively defined relationship exists between the lytic-latent switch and viral non-coding RNAs 3,4. Here, we identify miRNA-mediated inhibition of miRNA processing as a novel cellular mechanism that human herpesvirus 6A (HHV-6A) exploits to disrupt mitochondrial architecture, evade intrinsic host defense and drive the latent-lytic switch. We demonstrate that virus-encoded miR-aU14 selectively inhibits the processing of multiple miR30 family members by direct interaction with the respective pri-miRNA hairpin loops. Subsequent loss of miR-30 and activation of miR-30/p53/Drp1 axis triggers a profound disruption of mitochondrial architecture, which impairs induction of type I interferons and is necessary for both productive infection and virus reactivation. Ectopic expression of miR-aU14 was sufficient to trigger virus reactivation from latency thereby identifying it as a readily drugable master regulator of the herpesvirus latent-lytic switch. Our results show that miRNA-mediated inhibition of miRNA processing represents a generalized cellular mechanism that can be exploited to selectively target individual members of miRNA families. We anticipate that targeting miR-aU14 provides exciting therapeutic options for preventing herpesvirus reactivations in HHV-6-associated disorders like myalgic encephalitis/chronic fatigue syndrome (ME/CFS) and Long-COVID.

\section{Main Text}

MicroRNAs (miRNAs) are important regulators of gene expression that have been implicated in all major cellular processes of life ranging from embryonic development to tissue homeostasis and cancer 5,6 . Their biogenesis is tightly regulated at all levels ${ }^{7}$. Shortly after the discovery of cellular miRNAs, a number of viruses, predominantly of the herpesvirus family, were identified to encode and express their own set of viral miRNAs ${ }^{4,8}$. One of these is human herpesvirus $6 \mathrm{~A}(\mathrm{HHV}-6 \mathrm{~A})$, which has a seroprevalence of $>90 \%$ in the human population. HHV-6A establishes latency by integrating into the subtelomeric regions of host chromosomes ${ }^{9}$. Virus reactivation has been associated with cardiac dysfunction and graft rejection as well as neuronal disorders including myalgic encephalitis and chronic fatigue syndrome $(\mathrm{ME} / \mathrm{CFS})^{10}$. The later has recently sparked interest because of HHV-6A reactivations as a putative cause of ME/CFS-like symptoms observed in Long-COVID. Here, we reveal miRNA-mediated inhibition of miRNA processing as a novel cellular mechanism that HHV-6A exploits to disrupt mitochondrial architecture, evade the induction of type I interferons and facilitate virus reactivation from latency.

\section{HHV-6A induces mitochondrial fragmentation}

Mitochondria play a key role in the cell intrinsic defense against viruses. They constantly undergo fission and fusion events that help maintain functional mitochondria in cells under metabolic and environmental stress ${ }^{11}$. To examine whether HHV-6A affects mitochondrial architecture, we infected human umbilical vein endothelial cells (HUVEC) with wild-type HHV-6A and imaged mitochondria using a constitutively expressed, mitochondrially targeted GFP (mitoGFP) ${ }^{12}$. Lytic HHV-6A infection resulted in extensive 
mitochondrial fragmentation by 24 hours post infection (hpi) (Fig. 1a, Extended data Fig. 1a). The same was observed upon reactivation of latent HHV-6A in U2-OS bone osteosarcoma cells induced by Trichostatin-A (TSA) treatment (Extended data Fig. 1b). Mitochondrial fusion-fission dynamics are governed by the activity of Dynamin-related protein 1 (Drp1) ${ }^{13}$. Helical oligomers of Drp1 form a ring around the outer mitochondrial membrane and fragment it ${ }^{14}$. Mitochondrial fragmentation was reflected by increased Drp1 expression during both lytic HHV-6A infection (Fig. 1b) and virus reactivation (Extended data Fig. 1c) as well as colocalization of Drp1 on mitochondrial surfaces in the virusreactivated cells (Extended data Fig. 1d). Drp1 levels are directly controlled at transcriptional level by the p53 tumor suppressor protein ${ }^{15}$. Accordingly, both lytic HHV-6A infection and virus reactivation exhibited increased p53 expression (Fig. 1b, Extended data Fig. 1c) indicating that HHV-6A induces mitochondrial fragmentation via the canonical p53/Drp1 axis ${ }^{15}$.

\section{HHV-6A infection induces a miR-30 processing defect}

Human miR-30 family members regulate mitochondrial fusion and fission by targeting $p 53$ and its downstream target Drp1 ${ }^{12,15}$. Northern blots revealed a decrease in the expression of various miR-30 family members (miR-30a, miR-30c, miR-30d and miR-30e) upon lytic HHV-6A infection (Fig. 1c, Extended data Fig. 2a). Interestingly, loss of the most highly expressed miR-30c was accompanied by a concomitant increase in pri-miR-30c levels (Fig. 1d) indicating that HHV-6A infection affects pri-miR-30c processing. Similar results were also observed during TSA-induced HHV-6A reactivation (Extended data Fig. 2b-2c). Furthermore, small RNA sequencing data from HHV-6A infected HSB-2 T-cells confirmed the decrease in miR-30c and miR-30e levels (Extended data Fig. 2d).

\section{Viral miR-aU14 shares sequence homology to the miR-30c hairpin loop}

Unlike lytic infection, HHV-6A reactivation in the U2-0S cell model does not progress to fully productive virus replication but is restricted to the expression of some viral miRNAs and a few viral mRNAs ${ }^{16}$. We thus asked whether any of the HHV-6A small non-coding RNAs might be involved in the observed miR-30 processing defect. Manual sequence inspection revealed an interesting complementarity between HHV6A miR-U14 and the hairpin loops of pre-miR-30c, pre-miR-30a and pre-miR-30d (Fig. 1e, Extended data Fig. 3a). This viral miRNA is expressed at very high levels during both productive infection ${ }^{17}$ and virus reactivation ${ }^{16}$. However, it is encoded antisense to the U14 ORF. Hence, we decided to rename it as miRaU14. The miR-30c hairpin loop showed the strongest sequence complementarity to miR-aU14 (Extended data Fig. 3b-3c) ${ }^{18}$. Small RNA sequencing of Argonaute (Ago)-bound RNA from HHV-6A-infected HSB-2 cells confirmed this so far incompletely characterized viral miRNA to represent one of the two most abundant viral miRNAs in HHV-6A-infected cells (Extended data Fig. 4a). In addition, transcription start site profiling (TSS) using differential RNA-seq (dRNA-seq; Extended data Fig. 4b) ${ }^{19,20}$ identified the primiR-aU14 transcript, which initiates $158 \mathrm{nt}$ upstream of the miRNA (Extended data Fig. 4b) consistent with the unusual 130 nucleotide pre-miR-aU14 hairpin predicted by mFold ${ }^{21}$ (Extended data Fig. 4c). Of 
note, pre-miR-aU14 is highly conserved between HHV-6A and HHV-6B with the region complementary to the pre-miR-30c hairpin loop showing 100\% conservation (Extended data Fig. 4d).

\section{Viral miR-aU14 is sufficient to induce the miR-30c processing defect and mitochondrial fragmentation}

To assess the role of miR-aU14 in miR-30c processing, we employed a wild-type miR-aU14 miRNA mimic (Wt mimic) and a mutant thereof (Mut mimic) (Fig. 1f). Strikingly, transfection of the wild-type but not the mutant mimic into U2-OS cells reproduced both the loss of mature miR-30c and the concomitant increase in pri-miR-30c (Fig. 1g). To further validate that miR-aU14 was responsible for the miR-30c processing defect, we generated HeLa cells with a doxycycline (dox)-inducible miR-aU14 expressed from a Pol III promoter-driven shRNA and a mutant version (HeLa-Mut) thereof (Extended data Fig. 5a). Dox-induced expression of miR-aU14 (HeLa-Wt, Extended data Fig. 5b) but not of its mutant (HeLa-Mut, Extended data Fig. 5c) fully reproduced the miR-30c processing defect.

We next asked whether expression of miR-aU14 was sufficient to disrupt mitochondrial architecture via the p53/Drp1 axis. Interestingly, both transfection and dox-induced expression of miR-aU14 but not of the mutant thereof induced p53 and Drp1 expression (Extended data Fig. 5d, 5e). Furthermore, transfection of the miR-aU14 mimic fully recapitulated the mitochondrial fission phenotype (Extended data Fig. 5f).

To validate this effect in the virus context, we generated a mutant virus genome with discrete nucleotide substitutions within miR-aU14 (Extended data Fig. 6a). Mutations were designed not to alter the amino acid sequence of the U14 ORF. Deep sequencing of both the wild-type (HHV-6A-wt) and mutant (HHV-6Amut) bacterial artificial chromosomes (BAC) confirmed the introduced mutations to represent the only differences between the two viral genomes. In contrast to wild-type HHV-6A, we were unable to reconstitute the miR-aU14 mutant virus despite multiple attempts indicating that the loss of miR-aU14 severely reduced viral fitness. Hence, we generated polyclonal U2-OS cells that either carried chromosomally integrated latent wild-type HHV-6A or its miR-aU14 mutant by selection of cells stably transfected with the respective BACs. Upon virus reactivation with TSA, wild-type HHV-6A but not its miRaU14 mutant impaired pri-miR-30c processing (Fig. 1h), induced Drp1 expression (Extended data Fig. 6b) and triggered mitochondrial fission (Fig. 1i, Extended data Fig. 6c).

\section{Viral miR-aU14 inhibits miR-30 processing by direct RNA:RNA interaction}

To test whether miR-aU14 directly interacts with the pre-miR-30c hairpin loop, we affinity purified radioloabeled synthetic miR-aU14 with biotinylated pre-miR-30c. HeLa cells were transfected with equimolar amounts of radiolabeled synthetic miR-aU14, or two mutants thereof, and synthetic biotinylated pre-miR-30c (Fig. 2a, 2b). Radiolabeled RNA affinity purified $16 \mathrm{~h}$ later showed stronger enrichment of wild-type miR-aU14 than of its two mutants (Fig. 2c). In contrast, a radiolabeled small RNA control did not co-elute with pre-miR-30c.

We next asked whether the presence of the pre-miR-30c hairpin loop was sufficient to mediate its inhibitory effects on miRNA processing. We designed two artificial target pre-miRNAs that carried the 
original hairpin loop sequence of pre-miR-30c but contained artificial miRNA stem duplex sequences (termed: miR-A and miR-B) (Fig. 2d). We then generated polyclonal HeLa cells with dox-inducible Pol-IIIdriven expression of miR-A or miR-B as well as stable transduced with lentiviruses that express either wild-type or mutant miR-aU14 (as shown in Extended data Fig. 5a). Consistent with the predicted interaction of miR-aU14 with the pre-miR-30c hairpin loop, induction of miR-aU14, but not of the mutant thereof, strongly repressed both miR-A and miR-B processing (Fig. 2e, 2f).

\section{Viral miR-aU14 inhibits the induction of type I interferons}

Healthy mitochondria play an important role in intrinsic immunity ${ }^{22,23}$. Upon activation of toll-like or RIGI-like receptors, mitochondria serve as antiviral signaling hubs that govern the production of type I interferons (IFNs) ${ }^{24}$. RNA polymerase III can use cytosolic herpesvirus DNA as a template to produce 5'triphosphate RNAs, which induce type I IFN through the RIG-I pathway ${ }^{24-26}$. Enforced mitochondrial fission dampens RIG-I/MAVS signaling and reduces the induction of type I IFNs ${ }^{27}$. We thus asked whether miR-aU14-mediated mitochondrial fragmentation impacts on the induction of type I interferons. Exposure of HEK293T cells, transfected with the miR-aU14 mimic, to the R/G-/pathway activator 3phpRNA ( $5^{\prime}$ triphosphate hairpin RNA) ${ }^{28}$ resulted in reduced mRNA levels of IFNbeta (Fig. 3a) as well as the IFN-responsive IFIT1 gene (Fig. 3b) in comparison to cells transfected with the miR-aU14 mutant.

We next asked whether miR-aU14 also plays a role in suppressing the production of IFNbeta upon HHV$6 \mathrm{~A}$ reactivation. In addition to inducing virus reactivation by TSA, we treated cells with the JAK/STAT inhibitor Ruxolitinib to prevent secondary IFNbeta-mediated effects on virus reactivation. The latter was assessed by Northern blot for viral miR-aU14 and sncRNA-U77 (Fig. 3c). Ruxolitinib treatment enhanced TSA-induced virus reactivation resulting in a concordantly greater loss of miR-30c. Expression of viral sncRNA-U77 was significantly reduced for HHV-6A-mut indicative of impaired virus reactivation. Accordingly, miR-30c levels remained unchanged. Nevertheless, the mutant virus induced significantly greater levels of IFNbeta than the wild-type virus (Fig. 3d). This was further increased upon Ruxolitinib treatment, presumably due to the inhibition of secondary negative feedback loops of IFN signaling. While reactivation of wild-type virus reduced IFIT1 mRNA levels by $\sim 5$-fold, IFIT1 levels were increased by 1.2fold for HHV-6A-mut relative to non-reactivated cells. This was partially inhibited by Ruxolitinib treatment (Fig. 3e).

\section{Viral miR-aU14 governs the latent-lytic switch and augments productive infection}

Considering the observed effects of miR-aU14 on the induction of type I IFNs, we asked whether ectopic expression of miR-aU14 would augment productive wild-type virus infection and rescue reactivation of the mutant virus. Transfection of miR-aU14 but not of a control miRNA mimic significantly increased the number of cells productively infected with wild-type virus by $\approx 4$-fold (Fig. 3f). Furthermore, transfection of miR-aU14 efficiently rescued reactivation of the mutant virus even in the absence of TSA (Fig. $3 \mathrm{~g}$ ). The combination of both TSA and miR-aU14 showed enhanced virus reactivation indicating synergistic effects between the two. Similarly, transfection of miR-aU14 was substantially more effective at inducing 
reactivation of wild-type virus than TSA (Extended data Fig. 7a). Neither of the two mutant mimics had any effect on virus reactivation (Extended data Fig. 7b).

\section{Targeting hairpin loops and other unpaired sequences of human pre-miRNAs by small synthetic RNAs interferes with miRNA maturation}

In principle, miRNA-mediated inhibition of miRNA processing should be applicable to other cellular miRNAs. This is of particular interest as many important cellular miRNAs exist as miRNA families. Targeting hairpin loops rather than the mature miRNA sequences would offer a unique opportunity for the development of more selective miRNA inhibitors. Many of the let-7 family members carry relatively large hairpin loops, which may comprise up to $30 \mathrm{nt}$. Hence, we designed synthetic miRNA mimics targeting two different regions of the hairpin loop of pre-let-7d (Fig. 4a). Upon transfection into cells, both miRNA mimics efficiently reduced mature let-7d levels consistent with impaired miRNA processing (Fig. 4b). Similar data were obtained for two other miRNA mimics targeting the hairpin loop of let-7f1 (Extended data Fig. 8a, 8b).

Finally, we speculated that miRNA-mediated inhibition of miRNA processing should also be observable for cellular miRNAs. To identify such regulation, we carried out a systematic blast search of mature human miRNAs against known pre-miRNAs from miRBase. Several search results indicated interesting potential binding sites within pre-miRNAs (Extended data Table 1). However, majority of the respective miRNA pairs were not abundantly expressed in most of the standard human cell lines. We thus focused on one particular candidate pair of miRNAs, namely miR-155 and miR-148b. Our analysis indicated potential binding of miR-155 to pri-miR-148b just 5' of the pre-miR-148b hairpin (Fig. 4c). Transfection of a miR-155 mimic resulted in reduced levels of mature miR-148b. MicroRNA-mediated inhibition of miR$148 \mathrm{~b}$ processing by cellular miR-155 thus at least partially explains the dichotomous expression of these two human miRNAs ${ }^{29}$.

\section{Discussion}

Here, we identify miRNA-mediated inhibition of miRNA processing through sequence-specific RNA-RNA interactions as a novel cellular mechanism that governs miRNA processing (Extended data Fig. 9). Regulation of miRNA processing by cellular proteins is well described ${ }^{7}$. The first and best characterized example is the stem cell factor Lin-28, which interacts with the hairpin loop of let-7 family members and blocks their biogenesis ${ }^{30-32}$. Recently, a large unbiased screening approach identified $\sim 180$ RNA binding proteins that specifically interact with distinct human pre-miRNAs ${ }^{33}$. RBP-mediated regulation of miRNA processing thus constitutes an important regulatory network that shapes miRNA activity and function. Here, we show that miRNAs can take over similar functions and selectively inhibit miRNA processing in a sequence-specific manner. Interestingly, the miR-aU14-mediated loss of miR-30c was accompanied by a marked increase of only pri-miR-30c levels. This implies that the inhibition occurs at the level of primiRNA processing within the nucleus. miR-30c is encoded from an intron of the NF-YC gene ${ }^{34}$. Recognition and cleavage of the intronic pre-miRNA hairpin loop by the RNase II Drosha thus competes 
with the cellular splicing and RNA degradation machinery. Sterical interference of DGCR8 binding to the miR-30c hairpin by miR-aU14 in the nucleus and subsequent degradation of the parental intron upon splicing therefore is a likely explanation for the observed loss of mature miR-30c. In contrast, let-7d is expressed from its own primary transcript. Inhibition of let-7d processing by two artificial miRNAs targeting the hairpin loop indicates impaired pre-miRNA processing in the cytoplasm. We thus cannot exclude that miRNA-mediated inhibition of miRNA processing may occur both in the nucleus and cytoplasm. In addition to viral inhibition of miR-30, the master regulator of mitochondrial fusion and fission, we show that the inflammatory miR-1 $55^{35}$ inhibits pri-miR-148b processing. This explains previous reports of dichotomal expression of these two important human miRNAs ${ }^{29}$. The putative binding site of miR-155 within the pri-miR-148b closely flanks the pre-miR-148b stem. This implies that miR-155 binding disrupts the hairpin structure and thereby sterically inhibits pri-miR-148b cleavage by Drosha in the nucleus. We therefore propose a model that miRNAs can either sterically inhibit the binding of DGCR8 to the pri-miRNA hairpin loop or pri-miRNA cleavage by Drosha via base pairing with energetically favorable unpaired sequences in the respective pri-miRNAs. As we exemplify for two let-7 family members, miRNA-mediated inhibition of miRNA processing can be readily exploited to specifically target individual miRNAs of large miRNA families that so far could not be individually targeted.

Viral miR-aU14-mediated inhibition of miR-30 processing explained mitochondrial fragmentation during both lytic HHV-6A infection and virus reactivation via the miR-30/p53/Drp1 axis. This in turn impairs the induction of type I IFN and augments productive virus infection. Interestingly, multiple attempts to reconstitute a miR-aU14 mutant virus from BAC DNA failed, indicating that viral miR-aU14 is crucial for productive virus replication in vitro. Similarly, the miR-aU14 mutant virus was severely impaired in its ability to reactivate from latency. It is, however, important to note that reactivation of the miR-aU14 mutant virus by TSA resulted in a significantly stronger type I IFN response than observed for wild-type virus. This indicates that miR-aU14 may not be essential for the desilencing of the latent virus genomes but rather the inhibition of intrinsic cellular defense mechanisms that otherwise efficiently prevent successful virus reactivation. This is in line with the gross disruption of mitochondrial architecture by miR-aU14 via the miR-30/p53/Drp1 axis.

A peculiar feature of the miR-aU14 locus is that miR-aU14 is expressed antisense to the U14 open-reading frame from a novel pri-miRNA transcript that initiates within the front part of the U14 ORF. U14 encodes for a $\mathrm{G} 2 / \mathrm{M}$ cell cycle checkpoint regulator of HHV- ${ }^{36}$, which also interacts with $\mathrm{p} 53^{37}$. While miR-aU14 thus has the potential to repress expression of the important U14 protein, mutational analysis indicates that both U14 and miR-aU14 are important for productive HHV-6A infection. Accordingly, transient transfection of miR-aU14 enhanced productive HHV-6A infection and fully rescued virus reactivation of a miR-aU14 mutant virus. The most striking finding, however, was that transfection of miR-aU14 triggered virus reactivation from latency at least as efficiently as the commonly employed histone deacetylase inhibitor TSA. While enhanced mitochondrial fission and impaired intrinsic immunity via the miR30/p53/Drp1 axis will augment successful virus reactivation, miR-aU14 may also target other cellular or viral genes that help trigger virus reactivation from latency. 
In summary, our findings reveal a surprising, drugable miRNA-mediated mechanism that a prevalent human herpesvirus usurped to interfere with intrinsic immunity, govern the latent-lytic switch and augment productive infection. Importantly, viral miR-aU14 should be readily drugable using antisense approaches (antagomiRs) ${ }^{38}$, thereby providing an interesting therapeutic option for preventing herpesvirus reactivation in ME/CFS patients and Long-COVID.

\section{Declarations}

\section{Acknowledgements:}

We would like to thank the Core Unit for Confocal Microscopy and Flow Cytometry-based Cell Sorting of the IZKF Würzburg for supporting the study. Our thanks also goes to Prof. Markus Sauer and Dr. Suvagata Roy Chowdhury for their help with SIM microscopy and Prof. Thomas Rudel for his scientific support.

\section{Funding:}

We thank the Helmholtz Institute for RNA-based Infection Research (HIRI) for supporting this work with a seed grant to BKP through funds from the Bavarian Ministry of Economic Affairs and Media, Energy and Technology (Grant allocation nos. 0703/68674/5/2017 and 0703/89374/3/2017). LD is supported by the European Research Council (ERC-2016-CoG 721016 - HERPES). We also thank the HHV-6 Foundation, USA for Dharam Ablashi pilot research grant to BKP and the Solve ME/CFS Initiative, USA for the Ramsay Research Award to BKP.

\section{Author Contribution:}

BKP conceived the idea and carried out majority of the experiments; LD and BKP designed the project, and wrote the manuscript; $A B P, T H, A E, J T, A G, A W$ and FK contributed to miRNA intearaction studies and Interferon assay; $\mathrm{ML}$, carried out FACS analysis; $\mathrm{CJ}$, SH carried out dRNA-seq experiment and data analysis; BK, designed and developed the HHV-6A mutant BAC; FE, analyzed the sequencing data; $L D, G M$, analyzed the data. All the authors contributed to manuscript writing.

\section{Competing Interests:}

The authors declare that they have no competing interests.

\section{Data and Materials availability:}

Further information and requests for resources and reagents should be directed to Bhupesh K Prusty (bhupesh.prusty@uni-wuerzburg.de) and Lars Dölken (lars.doelken@uni-wuerzburg.de). The sequencing datasets produced in this study are deposited at GEO with the accession number GSE179867. (Reviewer access token: otkneyckdpyvdgp) 


\section{References}

1 Paludan, S. R., Bowie, A. G., Horan, K. A. \& Fitzgerald, K. A. Recognition of herpesviruses by the innate immune system. Nat Rev Immuno/ 11, 143-154, doi:10.1038/nri2937 (2011).

2 York, I. A. Immune evasion strategies of the herpesviruses. Chem Bio/ 3, 331-335, doi:10.1016/s1074-5521(96)90115-5 (1996).

3 Cohen, J. I. Herpesvirus latency. J Clin Invest 130, 3361-3369, doi:10.1172/JCI136225 (2020).

4 Pfeffer, S. et al. Identification of virus-encoded microRNAs. Science 304, 734-736, doi:10.1126/science.1096781 (2004).

5 Bushati, N. \& Cohen, S. M. microRNA functions. Annu Rev Cell Dev Bio/ 23, 175-205, doi:10.1146/annurev.cellbio.23.090506.123406 (2007).

6 Bartel, D. P. MicroRNAs: target recognition and regulatory functions. Cell 136, 215-233, doi:10.1016/j.cell.2009.01.002 (2009).

7 Bartel, D. P. Metazoan MicroRNAs. Cell 173, 20-51, doi:10.1016/j.cell.2018.03.006 (2018).

8 Cai, X. et al. Kaposi's sarcoma-associated herpesvirus expresses an array of viral microRNAs in latently infected cells. Proceedings of the National Academy of Sciences of the United States of America 102, 5570-5575, doi:10.1073/pnas.0408192102 (2005).

9 Kaufer, B. B. \& Flamand, L. Chromosomally integrated HHV-6: impact on virus, cell and organismal biology. Current opinion in virology 9C, 111-118, doi:10.1016/j.coviro.2014.09.010 (2014).

10 Agut, H., Bonnafous, P. \& Gautheret-Dejean, A. Laboratory and clinical aspects of human herpesvirus 6 infections. Clinical microbiology reviews 28, 313-335, doi:10.1128/CMR.00122-14 (2015).

11 Mishra, P. \& Chan, D. C. Metabolic regulation of mitochondrial dynamics. J Cell Bio/ 212, 379-387, doi:10.1083/jcb.201511036 (2016).

12 Chowdhury, S. R. et al. Chlamydia preserves the mitochondrial network necessary for replication via microRNA-dependent inhibition of fission. J Cell Biol 216, 1071-1089, doi:10.1083/jcb.201608063 (2017).

13 Smirnova, E., Griparic, L., Shurland, D. L. \& van der Bliek, A. M. Dynamin-related protein Drp1 is required for mitochondrial division in mammalian cells. Mol Biol Cell 12, 2245-2256, doi:10.1091/mbc.12.8.2245 (2001).

14 Kalia, R. et al. Structural basis of mitochondrial receptor binding and constriction by DRP1. Nature 558, 401-405, doi:10.1038/s41586-018-0211-2 (2018). 
$15 \mathrm{Li}, \mathrm{J}$. et al. miR-30 regulates mitochondrial fission through targeting p53 and the dynamin-related protein-1 pathway. PLoS genetics 6, e1000795, doi:10.1371/journal.pgen.1000795 (2010).

16 Prusty, B. K. et al. HHV-6 encoded small non-coding RNAs define an intermediate and early stage in viral reactivation. NPJ Genom Med 3, 25, doi:10.1038/s41525-018-0064-5 (2018).

17 Nukui, M., Mori, Y. \& Murphy, E. A. A human herpesvirus 6A-encoded microRNA: role in viral lytic replication. J Viro/ 89, 2615-2627, doi:10.1128/JVI.02007-14 (2015).

$18 \mathrm{Xu}, \mathrm{X}$., Zhao, P. \& Chen, S. J. Vfold: a web server for RNA structure and folding thermodynamics prediction. PLoS One 9, e107504, doi:10.1371/journal.pone.0107504 (2014).

19 Sharma, C. M. \& Vogel, J. Differential RNA-seq: the approach behind and the biological insight gained. Curr Opin Microbio/ 19, 97-105, doi:10.1016/j.mib.2014.06.010 (2014).

20 Whisnant, A. W. et al. Integrative functional genomics decodes herpes simplex virus 1. Nat Commun 11, 2038, doi:10.1038/s41467-020-15992-5 (2020).

21 Zuker, M. Mfold web server for nucleic acid folding and hybridization prediction. Nucleic Acids Res 31, 3406-3415, doi:10.1093/nar/gkg595 (2003).

22 Tiku, V., Tan, M. W. \& Dikic, I. Mitochondrial Functions in Infection and Immunity. Trends Cell Biol 30, 263-275, doi:10.1016/j.tcb.2020.01.006 (2020).

23 Kim, S. J., Ahn, D. G., Syed, G. H. \& Siddiqui, A. The essential role of mitochondrial dynamics in antiviral immunity. Mitochondrion 41, 21-27, doi:10.1016/j.mito.2017.11.007 (2018).

24 West, A. P. et al. Mitochondrial DNA stress primes the antiviral innate immune response. Nature 520, 553-557, doi:10.1038/nature14156 (2015).

25 Rasmussen, S. B. et al. Herpes simplex virus infection is sensed by both Toll-like receptors and retinoic acid-inducible gene- like receptors, which synergize to induce type I interferon production. $J$ Gen Viro/ 90, 74-78, doi:10.1099/vir.0.005389-0 (2009).

26 Samanta, M., Iwakiri, D. \& Takada, K. Epstein-Barr virus-encoded small RNA induces IL-10 through RIG-I-mediated IRF-3 signaling. Oncogene 27, 4150-4160, doi:10.1038/onc.2008.75 (2008).

27 Castanier, C., Garcin, D., Vazquez, A. \& Arnoult, D. Mitochondrial dynamics regulate the RIG-I-like receptor antiviral pathway. EMBO Rep 11, 133-138, doi:10.1038/embor.2009.258 (2010).

28 Hornung, V. et al. 5'-Triphosphate RNA is the ligand for RIG-I. Science 314, 994-997, doi:10.1126/science.1132505 (2006).

29 Liu, X. et al. MicroRNA-148/152 impair innate response and antigen presentation of TLR-triggered dendritic cells by targeting CaMKIlalpha. J Immuno/ 185, 7244-7251, doi:10.4049/jimmunol.1001573 
(2010).

30 Newman, M. A., Thomson, J. M. \& Hammond, S. M. Lin-28 interaction with the Let-7 precursor loop mediates regulated microRNA processing. RNA 14, 1539-1549, doi:10.1261/rna.1155108 (2008).

31 Rybak, A. et al. A feedback loop comprising lin-28 and let-7 controls pre-let-7 maturation during neural stem-cell commitment. Nat Cell Bio/ 10, 987-993, doi:10.1038/ncb1759 (2008).

32 Viswanathan, S. R., Daley, G. Q. \& Gregory, R. I. Selective blockade of microRNA processing by Lin28. Science 320, 97-100, doi:10.1126/science.1154040 (2008).

33 Treiber, T. et al. A Compendium of RNA-Binding Proteins that Regulate MicroRNA Biogenesis. Mol Cell 66, 270-284 e213, doi:10.1016/j.molcel.2017.03.014 (2017).

34 Patel, N., Tahara, S. M., Malik, P. \& Kalra, V. K. Involvement of miR-30c and miR-301a in immediate induction of plasminogen activator inhibitor-1 by placental growth factor in human pulmonary endothelial cells. Biochem J 434, 473-482, doi:10.1042/BJ20101585 (2011).

35 Alivernini, S. et al. MicroRNA-155-at the Critical Interface of Innate and Adaptive Immunity in Arthritis. Front Immuno/ 8, 1932, doi:10.3389/fimmu.2017.01932 (2017).

36 Mori, J. et al. Human Herpesvirus-6 U14 Induces Cell-Cycle Arrest in G2/M Phase by Associating with a Cellular Protein, EDD. PLoS One 10, e0137420, doi:10.1371/journal.pone.0137420 (2015).

37 Takemoto, M. et al. Human herpesvirus 6 open reading frame U14 protein and cellular p53 interact with each other and are contained in the virion. $J$ Viro/ 79, 13037-13046, doi:10.1128/JVI.79.20.1303713046.2005 (2005).

38 Sever, J. L. et al. Virus antibodies and multiple sclerosis. Arch Neuro/ 24, 489-494 (1971).

\section{Figures}


Fig. 1

a

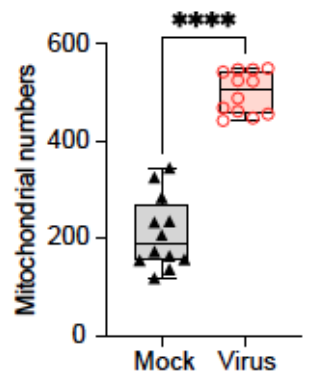

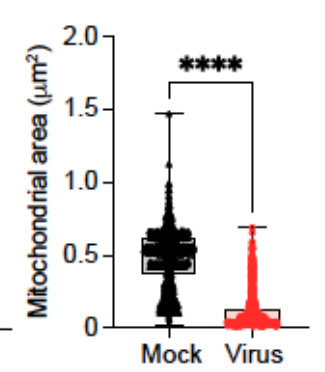

b

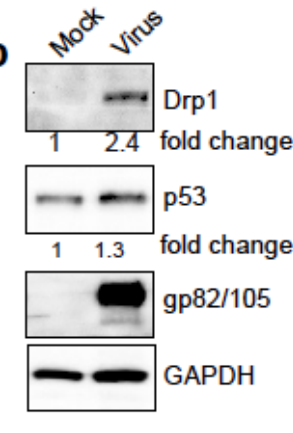

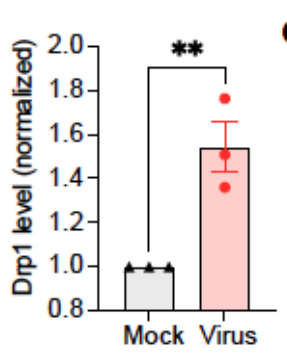

c

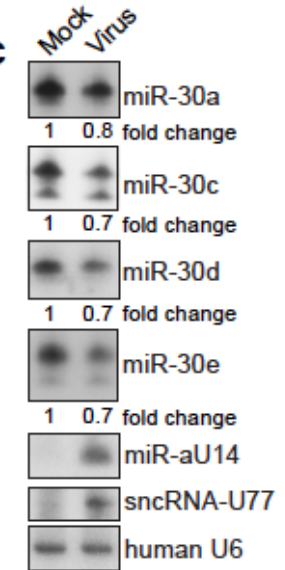

d

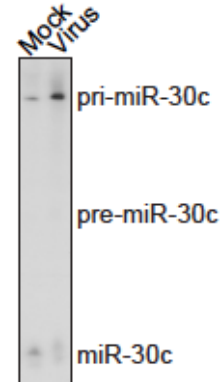

h human U6

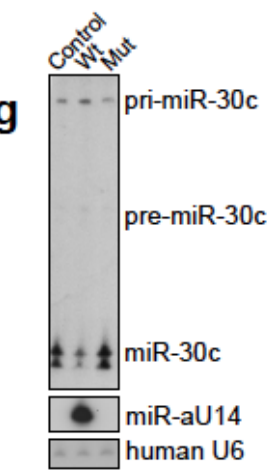

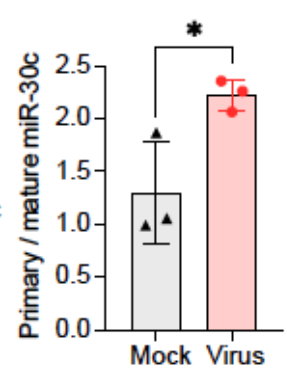

e

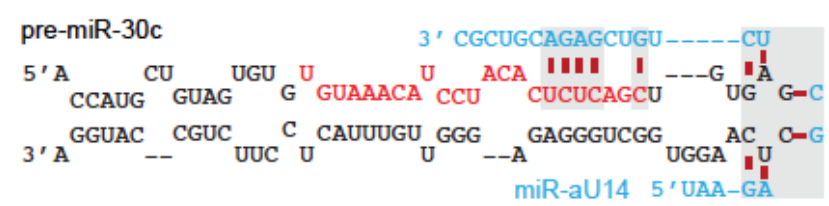

f miR-aU14 mimics UAAGAGCUCUGUCGAGACGUCGC Wt UaAGUGGAUUGUCGAGACGUCGC Mut
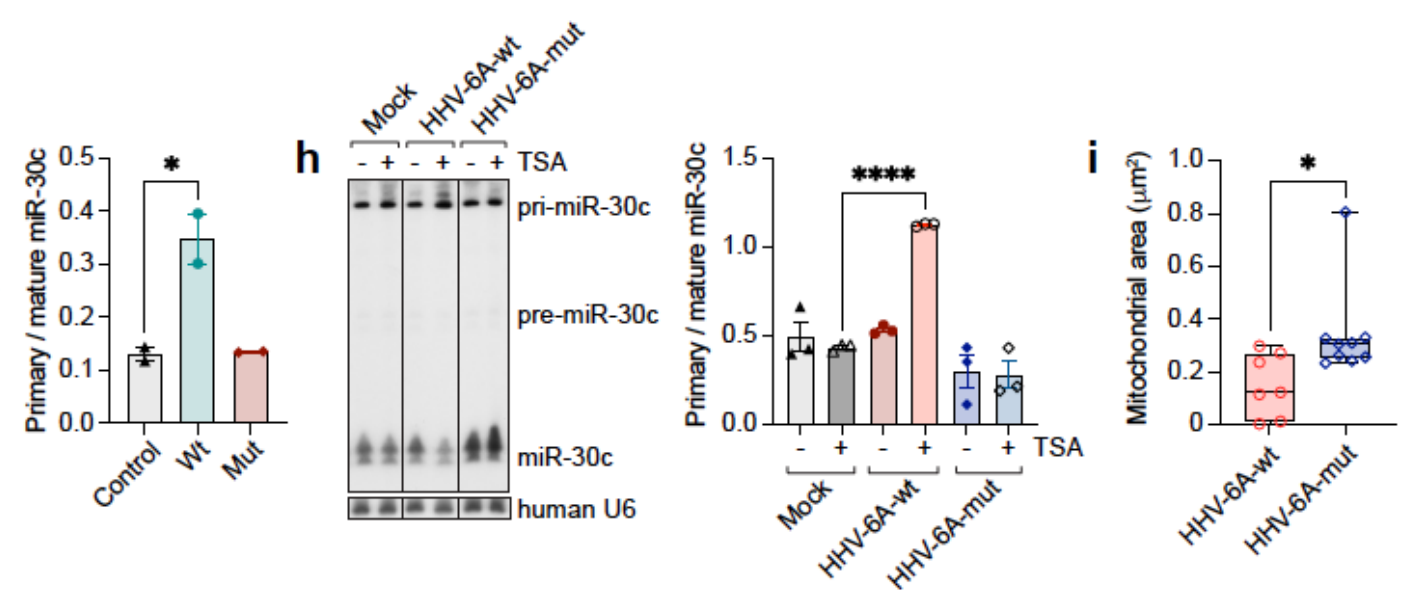

\section{Figure 1}

HHV-6A infection and reactivation induce mitochondrial fission via the p53/Drp1 axis. a. Mitochondrial numbers and average surface area in lytic HHV-6A infected primary HUVEC cells. Mitochondria from confocal images were quantified and are presented as box plots. $n=3$. b. p53 and Drp1 protein during mock (-HHV-6A) or HHV-6A (+HHV-6A) infection. HHV-6 infection was tested by viral glycoprotein gp82/105. GAPDH served as control. $n=3$. c. Mature miR-30 during lytic HHV-6A infection. HHV-6 infection was tested by viral miR-aU14 and sncRNA-U77. Human U6 served as control. d. miR-30c processing defect during lytic HHV-6A infection. $n=3$. e. Schematic of putative interaction of miR-aU14 with pre-miR-30c hairpin loop. Predicted sites of interaction are in grey boxes. f. Nucleotide sequences of wild-type (Wt mimic) and mutant (Mut mimic) miR-aU14 mimics. Point mutations are highlighted in blue 
within red boxes. g. Pri-miR-30c processing defect by wild-type or mutant miR-aU14 mimic. $n=2$. h. PrimiR-30c processing defect during reactivation of wild-type HHV-6A (HHV-6A-wt) or mutant virus (HHV-6Amut). U2-OS cells without HHV-6A served as mock control. $n=3$. i. Average mitochondrial area in wild-type or mutant HHV-6A reactivating cells as a box plot. RNA and protein quantification was done by densitometric analysis $(b, d, g, h)$. Box and whiskers $(a, i)$ show minimum to maximum values with all independent replicates, centre denotes median, and the bounds denote the 25th to 75th percentiles. Data are mean \pm s.e.m. ${ }^{*} P \leq 0.05,{ }^{\star *} P \leq 0.005,{ }^{\star *}+P \leq 0.00005$, two-way ANOVA with Tukey's multiple comparisons test $(a, f)$, unpaired two-tailed Student's t-test $(b, d, g, h)$.

Fig. 2

a

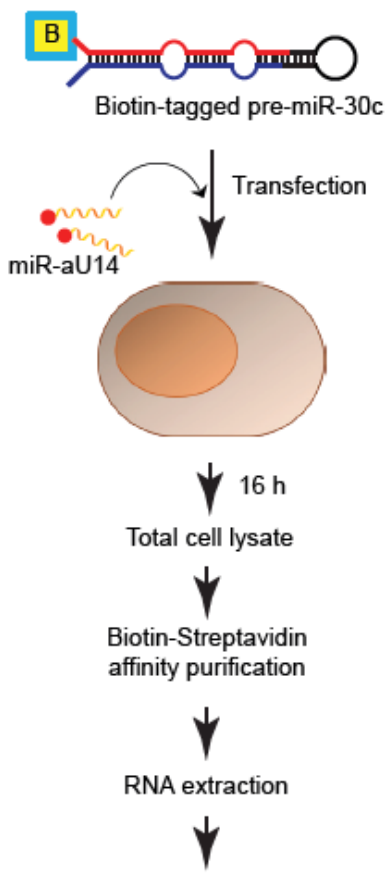

Northern hybridization b UAAGAGCUCUGUCGAGACGUCGC Wt mimic
UAAG GAUUGUCGAGACGUCGC Mut mimic
UAA CUCGAUUGUCGAGACGUCGC Mut2 mimic

C $\quad$ - - + + Control mimic

- - + Mut2 mimic

-+- Mut mimic

$\mathrm{DM}+\ldots$ - Wt mimic

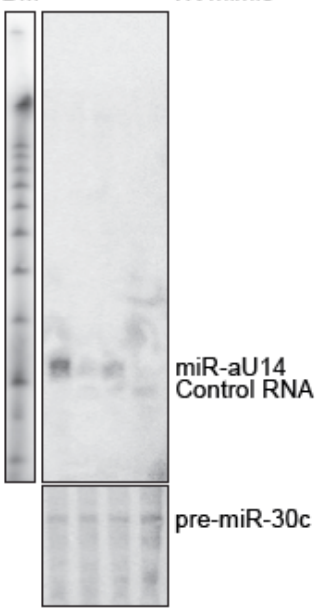

e

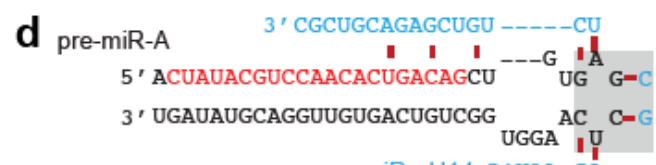

miR-aU14 5'UAA-GA
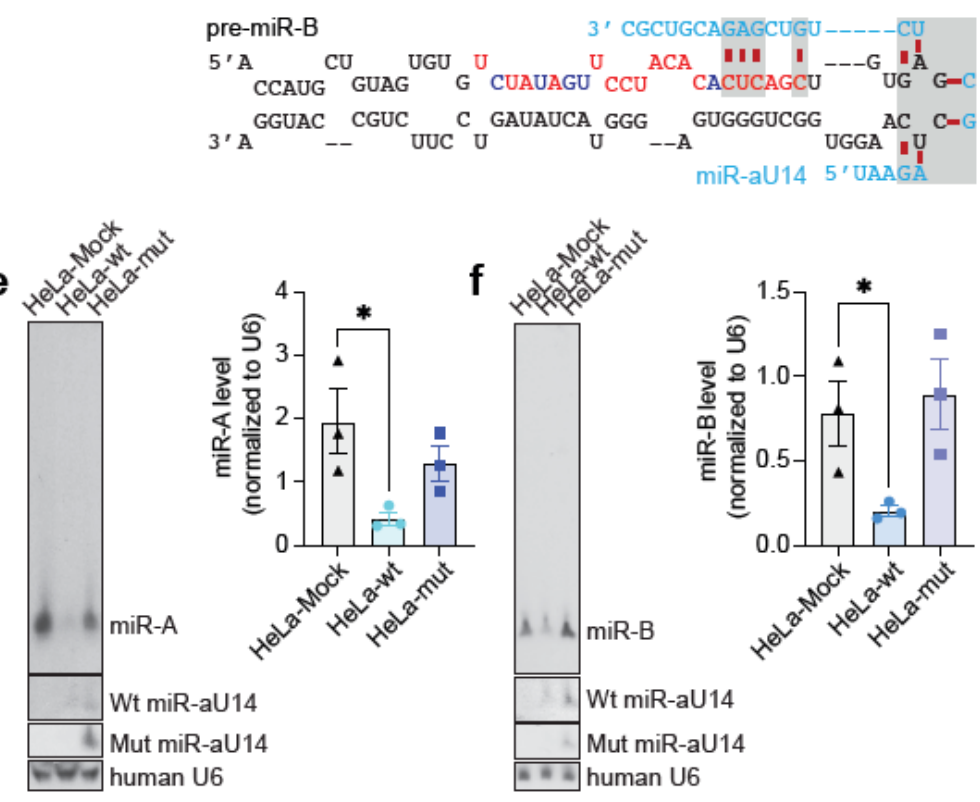

\section{Figure 2}

HHV-6A miR-aU14 inhibits miR-30 processing by direct RNA:RNA interaction a. Schematic of the experimental set up. b. Nucleotide sequences of the wild-type miR-aU14 mimic (Wt mimic) and the mutant mimics (Mut and Mut2 mimic). Mutated sequences are in blue within the rectangular boxes. c. Affinity purification of biotinylated pre-miR-30c co-precipitates miR-aU14 as in Fig. 2a. A random small RNA was used as a bait for control. The blot was probed for miR-30c for equal amounts of affinity purified biotinylated pre-miR-30c. d. Putative interactions of the two artificial chimeric pre-miRNAs (premiR-A and pre-miR-B) with miR-aU14. The mature miRNA sequences (miR-A and miR-B) are indicated in red. The putative interactions of the transplanted miR-30c hairpin loop with miR-aU14 (blue) are indicated. e, f. miR-aU14 impairs processing of artificial miRNAs carrying miR-30c hairpin loop. 
Polyclonal HeLa cells stably transduced for dox-inducible expression of miR-A or miR-B were retransduced either with wild-type miR-aU14 (HeLa-Wt), mutant miR-aU14 (HeLa-Mut) or with an empty vector (HeLa-Mock). $n=3$. Data are mean \pm s.e.m. ${ }^{*} P \leq 0.05$, two-way ANOVA with Tukey's multiple comparisons test $(e, f)$.

\section{Fig. 3}
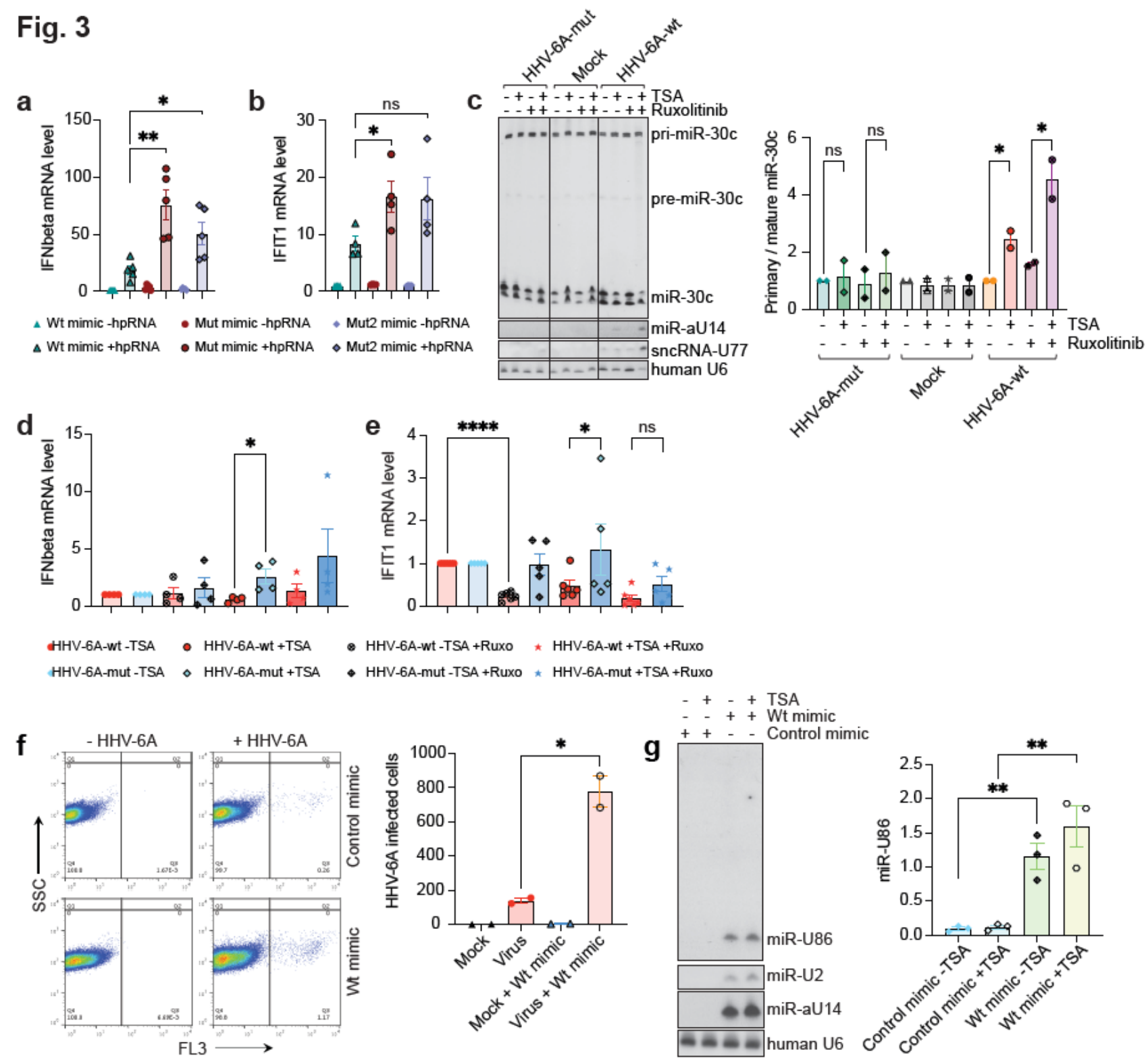

\section{Figure 3}

miR-aU14 induced mitochondrial fragmentation suppress induction of interferon beta. a. Relative IFNbeta mRNA levels in hpRNA stimulated cells in presence of either miR-aU14 mimic (Wt) or a mutant mimic (Mut) as compared to unstimulated cells. qRT-PCR values are normalized to 5S RNA. $n=5$. b. IFIT1 mRNA as in Fig. 3a. c. Pri-miR-30c processing defect upon reactivation of wild-type HHV-6A (HHV-6A-wt) or the miR-aU14 mutant (HHV-6A-mut) by TSA alone or together with Ruxolitinib. Cells without HHV-6A served as mock control. Virus reactivation was studied from fmiR-aU14 and sncRNA-U77. $n=3$. d. Relative 
IFNbeta mRNA levels from the experiment as in Fig. 3c. e. Relative IFIT1 mRNA levels from the experiment as in Fig. 3c. f. Lytic virus infection in presence of miR-aU14. HSB-2 cells were transfected with either a control mimic or the miR-aU14 mimic. HHV-6A mCherry reporter virus-was used to measure cell to cell spread of virus infection, as measured by flow cytometry. $n=2$. g. U2-OS cells carrying latent mutant HHV6A miR-aU14 (HHV-6A-mut) were transfected with either the miR-aU14 mimic or a control mimic. Cells were induced with TSA and the extent of virus reactivation was analyzed from viral miR-U2 and miR-U86. Human U6 normalized miR-U86 levels are presented as a bar diagram. $n=3$. Data are mean \pm s.e.m. $n s, P$ $>0.05, * P \leq 0.05,{ }^{*} \mathrm{P} \leq 0.005,{ }^{\star * \star *} \mathrm{P} \leq 0.00005$, two-way ANOVA with Tukey's multiple comparisons test (c, g, e), unpaired two-tailed Student's t-test (d, f).

\section{Fig. 4}

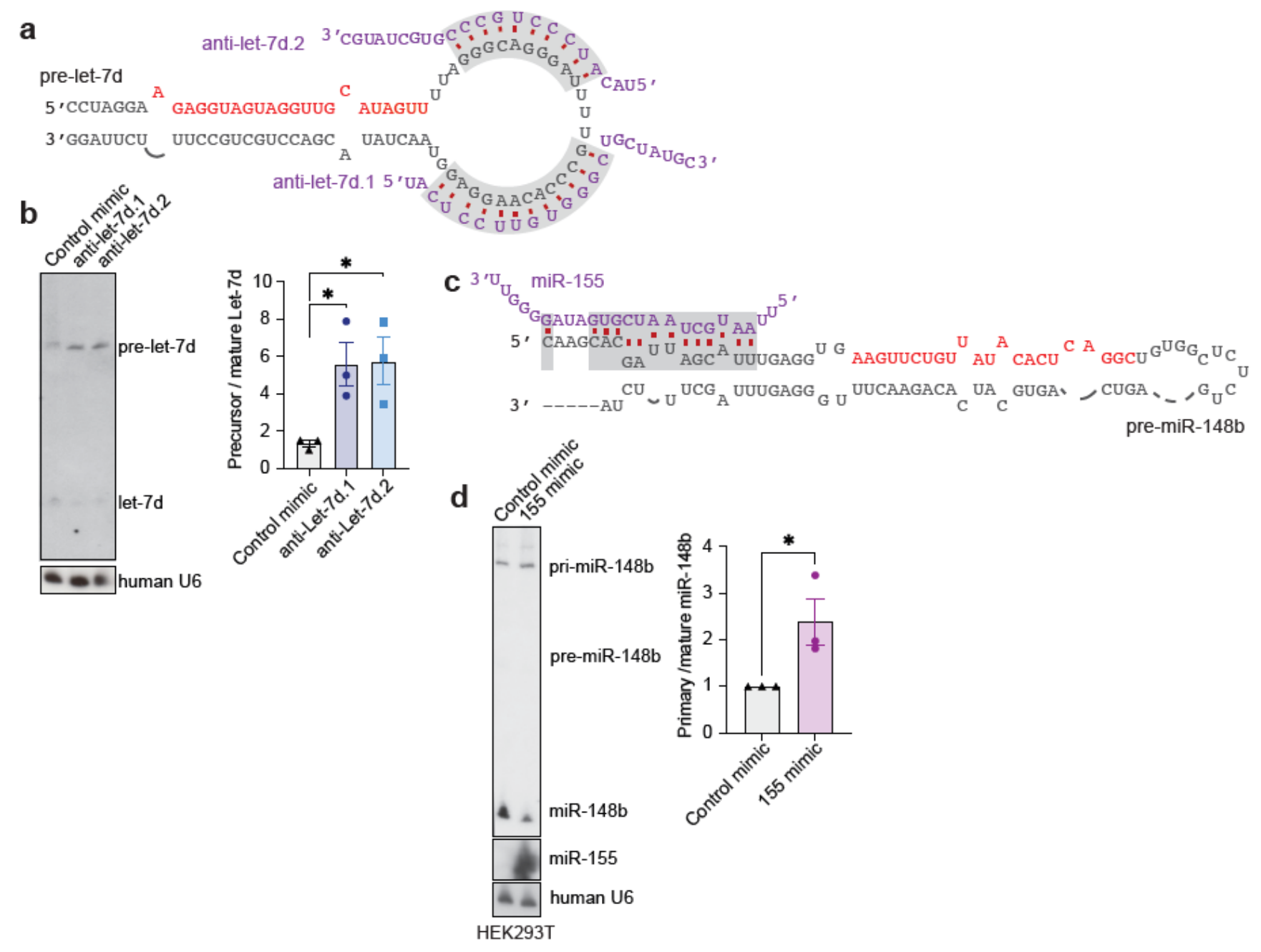

\section{Figure 4}

Human miRNA processing can be selectively inhibited by synthetic small RNAs. a. Schematic of putative binding of two different synthetic miRNAs to human pre-let-7d. b. miR-148b expression in U2-OS cells transfected with miRNA mimics designed against the hairpin loop of pre-let-7d were analyzed by Northern 
blotting. Precursor/mature let-7d ratios, as determined by densitometry analysis, are shown as bar diagram. $n=3$. c. Schematic of the putative binding of miR-155 to pri-miR-148b. Possible sequence interactions between the two RNAs are highlighted in grey boxes. $\mathrm{d}$. miR-148b expression in presence of synthetic miR-155 mimic. Primary/mature miR-148b, as determined by densitometry analysis, are shown as bar diagram. $n=3$. Data are mean \pm s.e.m. ${ }^{*} P \leq 0.05$, two-way ANOVA with Tukey's multiple comparisons test (b), two-tailed Mann-Whitney U-test (d).

\section{Supplementary Files}

This is a list of supplementary files associated with this preprint. Click to download.

- SupplementaryFigures.pdf

- SupplementaryTable1.pdf

- SupplementaryTable2.pdf

- ExtendeddataFigures19.pdf

- ExtendeddataTable1.pdf 